\title{
Mapping trajectories of Pgp-1 membrane protein patches on surfaces of motile fibroblasts reveals a distinct boundary separating capping on the
}

\section{lamella and forward transport on the retracting tail}

\author{
BRUCE F. HOLIFIELD ${ }^{1, *}$ and KEN JACOBSON ${ }^{1,2}$ \\ ${ }^{1}$ Department of Cell Biology and Anatomy and ${ }^{2}$ Lineberger Cancer Research Center, University of North Carolina at Chapel Hill, \\ Chapel Hill, NC 27599, USA \\ * Present address: Department of Cell Biology, Baylor College of Medicine, Houston, TX 77031, USA
}

\begin{abstract}
Summary
Patches of aggregated membrane proteins on motile fibroblasts are transported from the surfaces of the leading lamella to a site just ahead of the nucleus in the phenomenon known as capping. A major cell surface glycoprotein, Pgp-1 (GP80), was tagged with a monoclonal IgG and then aggregated with fluorescent secondary antibodies. Correlated digitized fluorescence and phase-contrast microscopy were used to map the trajectories of fluorescent Pgp-1 patches located in various regions of the cell surface. The response of patches located in lamellar and nonlamellar regions to spontaneous retraction of the trailing cell margin, or tail was examined in detail. During capping, Pgp-1 patches accumulated at a morphologically distinct site on the cell surface, the 'null border', corresponding to the boundary between lamelloplasm and endoplasm and the posterior edge of the dorsal cortical F-actin sheath. Posterior to this site, gradual forward movement of patches accompanied the gradual narrowing phase of the trailing edge retraction that occurs prior to abrupt detachment of the tail, but patches did not actually accumulate at the null border. The rate of
\end{abstract}

forward patch movement was generally greater at positions further behind the boundary. Patch movement correlated approximately with forward organelle movement in the trailing region of the cell. The boundary was also apparent during simultaneous capping and retraction when forward patch transport on the trailing edge and rearward transport of patches across the lamellar surface appeared to converge on the null border. Forward patch transport was strictly confined to regions behind the boundary while retrograde patch transport was confined to the lamellar region ahead of the boundary. Patches are thought to be linked to the cortical cytoskeleton and their transport is discussed in terms of the very different cortical cytoskeletal dynamics occurring in the leading and trailing edges of locomoting cells.

Key words: cell surface, cortical cytoskeleton, cell locomotion, capping, plasma membrane protein, $\mathrm{Pgp}-1 / \mathrm{CD} 44$, video microscopy

\section{Introduction}

Detailed analyses of cell motile activities in vitro have resulted in descriptions of several types of rearward directed movements of cellular structures and surfaceadsorbed material. Particles of various materials adsorbed to the plasma membrane of protrusive cell margins are moved rearward relative to both the substratum and the cell body (Abercrombie et al. 1970b; Harris and Dunn, 1972; Fisher et al. 1988). Lamellipodia and filopodia arise at or near such cell margins and also move rearward (Abercrombie et al. 1970a; Harris, 1973). Highly dynamic cytoskeletal structures, composed in part of F-actin and described as arcs, arise near the cell margins and move centripetally (Sorrano and Bell, 1982; Heath, 1983a). Cross-linked plasma membrane proteins are redistributed from lamellar surfaces in a manner similar to particle transport (Abercrombie et al. 1972; Edidin and Weiss, 1972; Vasiliev et al. 1976; Heath, 1983b). These rearward transport phenomena are not displayed by non-motile cells

Journal of Cell Science 98, 191-203 (1991)

Printed in Great Britain (C) The Company of Biologists Limited 1991 and thus are considered to be related to the complex molecular interactions involved in generating the tractive forces involved in cell locomotion (Harris, 1973).

Information concerning the relative movements of discrete points on the cell surface has come largely from studies of particle transport. The motion consists of a Brownian component in addition to a directed component (Dembo and Harris, 1981; Sheetz et al. 1989). However, direct information concerning movement of defined membrane proteins is lacking. On the other hand, the transport of antibody-induced aggregates of defined membrane proteins, the phenomenon called 'capping' on lymphocytes (Taylor et al. 1971), has not previously been exploited as a means of tracking movements of discrete identified plasma membrane components. This is due primarily to the limitations of the microscopy techniques used to visualize the protein aggregates, or patches. Typically, surface glycoproteins have been tagged with fluorescent ligands, and differences in initial and final ligand distributions within a cell population have been recorded photographi- 
cally. Continual recording of the dynamic distribution of patches on an individual cell was precluded by fluorescence fading and phototoxic effects associated with prolonged excitation of the fluorescent probes. Although several studies of cell surface transport phenomena have used image intensification and video techniques to surmount these problems (Koppel et al. 1982; Heath, $1983 b$ ), movements of individual identified patches were not resolved in either of these studies. More recently Webb and Gross (1986) were able to track movements of individual receptors using highly fluorescent low-density lipoprotein particles.

In this paper we describe results from tracking the lateral transport of discrete identified patches of a defined membrane protein on motile fibroblasts. The patches consist of aggregates of Pgp-1 (or GP80), a major murine plasma membrane glycoprotein (Hughes and August, 1981; Trowbridge et al. 1982). It has been cloned and sequenced and has a 266 residue ectodomain, a single pass transmembrane domain, and a 72 residue cytoplasmic domain (Wolffe et al. 1990). Mature Pgp-1 exhibits an $85 \times 10^{3}$ apparent relative molecular mass, the end result of a number of processing steps (Wolffe et al. 1990). The human homolog is the CD44 cell surface antigen, a collagen binding receptor (Carter and Wayner, 1988), which shows cell-cell recognition properties and functions as a lymphocyte homing receptor (for review, see Stoolman, 1989). The finding that several hemopoetic cells and cell lines expressing CD44 (Pgp-1) can be induced to aggregate by exposure to monoclonal antibodies directed against this antigen (Belitsos et al. 1990) suggests a role for CD44 as a mediator of cell-cell adhesion.

Earlier, Vasiliev et al. (1976), emphasizing the lamellar region of fibroblasts, showed that in the lectin-induced capping of surface receptors, the cleared zone terminated at a perinuclear boundary ahead of the nucleus. Focussing on the tail of motile fibroblasts, Chen (1981a) later showed that, upon retraction, particles attached to the trailing edge surface and internal organelles also moved toward a perinuclear boundary he termed the 'null border'. In this study, we extended this earlier work by mapping the trajectories of Pgp-1 patches during the simultaneous processes of capping and retraction on motile fibroblasts. Our studies show that patches are transported rearward across the lamellar surface, accumulating at a distinct boundary. This surface is underlain by an intricate cortical F-actin sheath. This sheath is dynamic as shown by the rearward transport of patches in concert with the retrograde movement of F-actin 'arcs'. Patches on the retracting trailing region of the cell surface are simultaneously transported forward towards the same boundary but do not undergo a net accumulation at the boundary, suggesting a folding or elastic recoil of the cell surfacecortical cytoskeleton in this region. Thus, patches on the cell surface are localized into two discrete regions, characterized by different cortical cytoskeletal properties. Furthermore, the boundary maintains the integrity of these two surface regions as patches from either region do not intermingle as the cell translocates across a substratum.

\section{Materials and methods}

Cells and fuorescent labeling protocols

The C3H 10T1/2 cell line was obtained from American Type Culture Collection (Rockville, MD) and was cultured in Eagle's basal medium with Earle's salts supplemented with $5 \%$ fetal bovine serum and $10 \mathrm{~mm}$ Hepes. Cells were seeded onto $22 \mathrm{~mm}$ coverslips and used $24-48 \mathrm{~h}$ after plating. Anti-Pgp-1 monoclonal IgG was affinity purified as previously described (Holifield et al. 1990). Stack solution of purified antibody in phosphate-buffered saline was diluted into warmed growth medium to a concentration of $50-100 \mu \mathrm{g} \mathrm{ml}^{-1}$. Cells on coverslips received $20-30 \mu \mathrm{l}$ of this solution for 1-2 min at room temperature, then were washed twice with $200 \mu \mathrm{l}$ of warmed growth medium. Rhodamineconjugated rabbit anti-rat IgG secondary antibody was then applied for $1 \mathrm{~min}$ at room temperature, followed by two rinses with warmed growth medium. Coverslips with cells were mounted into an aluminium observation chamber either prior to or immediately following application of antibodies.

Cells to be stained for F-actin were permeabilized in $0.1 \%$ Triton X-100 for $5 \mathrm{~min}$ at room temperature after fixation in $3.7 \%$ formaldehyde in PBS and a 1:50 dilution of the stock solution of rhodamine-phalloidin (Molecular Probes, Junction City, OR) was applied to each coverslip for $30 \mathrm{~min}$ at room temperature. Coverslips were then washed three times with PBS and mounted.

\section{Microscopy}

The observation chamber was mounted onto the stage of either a Zeiss IM-35 or Nikon Diaphot inverted microscope and was maintained at approximately $37^{\circ} \mathrm{C}$ by an air curtain incubator. A Dage model 66 (Dage, Inc., Michigan City, IN) intensified silicon intensifier target (ISIT) camera was employed to record fluorescence images while a Dage model 77 chalnicon video camera was used to record phase-contrast images. Images were acquired and digitized using laboratory microcomputers as previously described (Ishihara et al. 1988; DiGuiseppi et al. 1985).

\section{Photography}

Fixed and labelled cells were mounted onto coverslips using Gelvatol mounting medium for $35 \mathrm{~mm}$ photography. Fluorescence photomicrographs were taken with an Olympus OM-2 camera using Kodak Tri-X 400 film and Diafine developer. Digital images were printed electronically using a Sony color video printer or were photographed directly from the video monitor with the same film and developer described above.

\section{Image processing}

Both fluorescence and phase-contrast images were improved by background subtraction. Drift of the specimen field was corrected by image alignment based on the initial position of a stationary substratum marker. This was accomplished by viewing sequential stored images in two frame buffers and shifting one relative to the other.

\section{Tracking patch movements}

Analysis of individual patch movements used images stored in computer memory. During an experiment we collected sequentia sets of high-magnification fluorescence and phase-contrast images at approximately 3-min intervals. Each image set, corresponding to one time point, consisted of a phase-contrast image and two fluorescence images taken at two focal planes. Labelled cells were selected for tracking experiments on the basis of three criteria: (1) the surface density of patches was sufficiently low for unambiguous identification of individual patches to be possible; (2) patches appeared as discrete structures when sharply in focus at the onset of observation; (3) relatively broad surface areas could be focused at two focal planes.

These criteria were developed by trial and error during attempts to select cells randomly for patch tracking. In most cases this selection process led us to examine cells that capped patches more slowly than the majority of cells. On cells that capped most rapidly, patches tended to coalesce into clusters in which the individuality of patches was lost, precluding identification of a given patch at each time point. We recorded patch speeds of $0.2-0.5 \mathrm{~km} \mathrm{~min}{ }^{-1}$, for the cells that capped more slowly, which is in the lower range of reported capping rates. (In a given cell population, a range of capping rates exists that is presumably related to variations in the overall motile states of individual 
cells.) We also found that size and density of patches varied noticeably among cells in a population and frequently precluded unambiguous identification of individual patches. Because there is no a priori basis for a unit patch size, the individuality of a patch was defined operationally when a particular patch moved as a single structure without fusing with neighbors or fragmenting (these latter events did accur infrequently).

A computer program for extraction of $x y$ pixel coordinates from digital images displayed on a video monitor was written in Forth code (Uniforth Software Systems, Columbus, $\mathrm{OH}$ ) with some assembler calls for more rapid frame buffer accesses, and was menu-driven and batch-oriented. Either the ventral or dorsal fluorescence image from an image set was displayed on command. A patch to be tracked was identified by the user. A cursor was moved to the center pixel of the patch, and those pixel coordinates were written to a data file. This file was structured as a number matrix, with the row and column pointers automatically linked to the current time point and patch pointer values. Stored coordinates were recalled from the data file to reconstruct patch trajectories graphically. From the $x, y$ coordinates we determined component displacements in pixel units. Real displacements were calculated by imaging a stage micrometer and taking into account the display aspect ratio of 3 to 4 . From real displacements and clock time stored in image headers, patch velocities for each time interval were calculated and written to the data file.

Rapid display of images from four adjacent time points simulated time-lapse patch motion and facilitated identification of the same patch in successive images. When the identification was not immediately apparent, comparison of patch distributions at two focal planes permitted distinction between patches located on the dorsal and the ventral cell surfaces. Owing to the thinness of the cell at its periphery, patches on both the dorsal and ventral surface could be in focus in the lower focal plane at the leading cell margin. As patches moved rearward up the inclined dorsal surface, they moved out of focus in the lower plane and into focus at the higher plane. Focal planes were selected manually. If the new position of a patch could not be identified with certainty, then further tracking of that patch was stopped. All identifiable
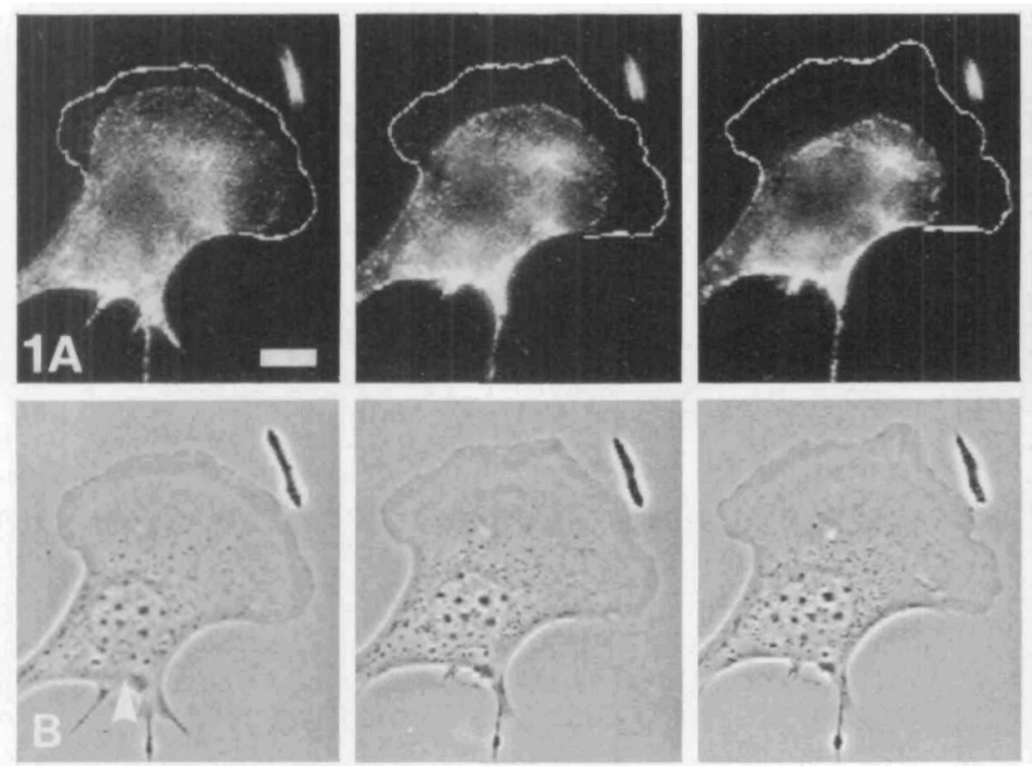

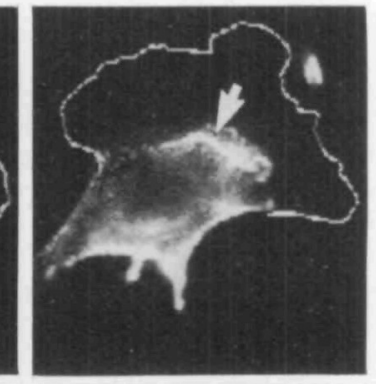

patches in a data set were tracked in at least two separate analysis sessions to safeguard against misidentification.

\section{Results}

\section{Capping on motile fibroblasts is a regionalized transport process}

We focused our attention on cells that had a morphology similar to the cells shown in Figs 1 and 2. This can be described as unipolar, unilamellar or tailed morphology. This is characteristic of motile fibroblasts and is a transient morphology, being one phase in a continual cycle of morphological changes involving leading edge extensions, cell elongation and trailing edge, or tail, retraction (Abercrombie et al. 1977; Dunn, 1980; Chen, 1981a,b). Selection of such cells for study is valid, since many of the cells in a population assume this morphology at some time during locomotion. Comparison of cells having similar morphologies is also helpful in discerning and describing cell surface movements, which are general features of cell locomotion.

The general features of patch clearance, or capping, on fibroblasts are illustrated in Fig. 1. Patches of Pgp-1 were formed throughout the cell surface within minutes after applying monoclonal IgG anti-Pgp-1 and then the aggregating anti-IgG. During a period of $30 \mathrm{~min}$, the cleared zone became broader as patches accumulated at a site just anterior to the nucleus, indicated by the increase in patch density at that site (see arrow, Fig. 1). Note that portions of the anterior cell margin advanced only a small distance forward and that the posterior cell margin progressively retracted during this time.

The site of patch accumulation is shown in more detail in Fig. 2. Shown are a phase-contrast image and fluor-
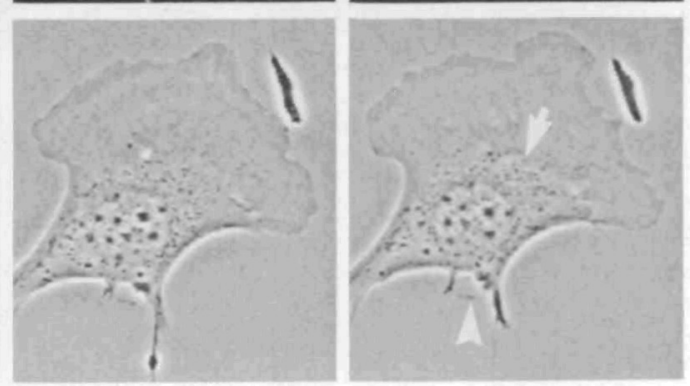

Fig. 1. General features of capping on motile fibroblasts. This sequence begins $5 \mathrm{~min}$ after applying anti-Pgp-1 mAb followed by rhodamine-conjugated secondary antibody to cells mounted in a chamber on the microscope stage. Patches of aggregated Pgp-1 had formed throughout the cell surface and a peripheral zone $5 \mu \mathrm{m}$ wide had already been cleared of patches. Such rapid clearance of this peripheral zone usually occurred in less than $2 \mathrm{~min}$, before excess fluorescent $\mathrm{Ab}$ was washed completely from the chamber. Time points shown are 5, 14, 28 and $40 \mathrm{~min}$ after applying $\mathrm{mAb}$. The cleared zone becomes broader as fluorescent patches accumulate at a site just anterior to the nucleus (arrowg). White outlines in fluorescent micrographs in series $\mathrm{A}$ (and in subsequent figures) delineate leading cell margins obtained from companion phase-contrast micrographs in series B. During the clearance of patches from the approximately $30 \mu \mathrm{m}$ wide lamellar region, the advance of the leading cell margin is only several micrometers, showing that patches move relative to both the cell and the substratum. Note also that the posterior or trailing cell margin gradually retracts forward during this time period (compare arrowheads in B). Bar, $10 \mu \mathrm{m}$. 

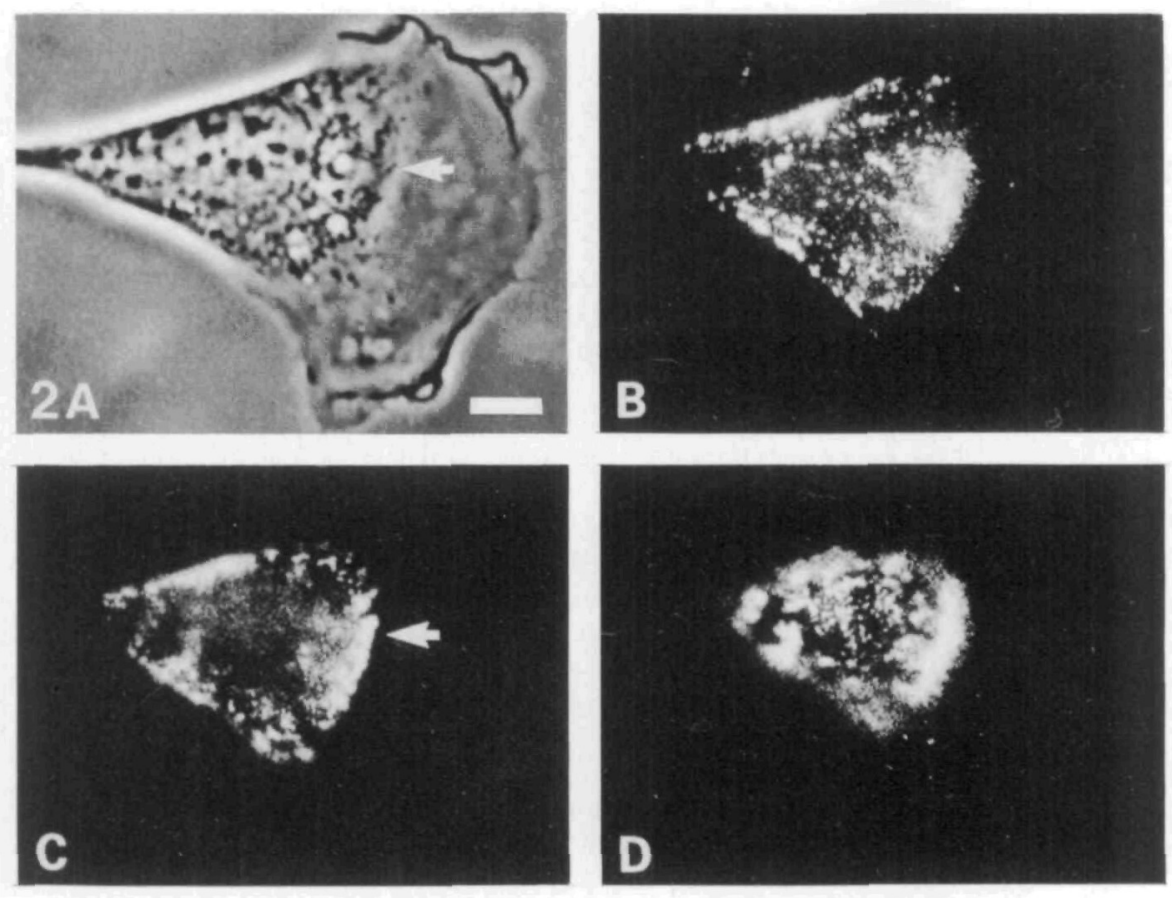

Fig. 2. Patches accumulate at a morphologically distinct site. This cel was incubated for approximately 30 min after patching Pgp-1. A digitized phase-contrast image is shown in $A$, and a through-focus series of digitized fluorescence images are shown: (B) focus is at the substratum (C) focus is at the site of patch accumulation anterior to the nucleus (arrow); (D) focus is at the cell apex over the nucleus. Patches accumulated on the dorsal surface at the site corresponding to the demarcation between the anterior lamelloplasm and the posterior endoplasm (compare arrows in $\mathrm{A}$ and $\mathrm{C}$ ). After accumulating at such a site, patches do not continue rearward and capping is completed. Bar, $10 \mu \mathrm{m}$. escence images taken at dorsal, intermediate and ventral focal planes $30 \mathrm{~min}$ after patch formation. Patches had accumulated on the dorsal surface (Fig. 2C) at a site corresponding to the demarcation between an anterior region having relatively few visible vesicular organelles and a posterior region having a high density of perinuclear organelles (arrow, Fig. 2A). Patches located posterior to this site did not move rearward and, once accumulated at such a site, patches stopped their rearward directed motion. This was confirmed by observing the cell in Fig. 2 at higher magnification for an additional $30 \mathrm{~min}$ (data not shown). However, posterior to the site of patch accumulation, a gradual forward movement of patches occurred, which correlated with slight retraction of the tail. Such movements are examined below in detail.

Vasiliev et al. (1976) introduced a terminology that is conceptually useful, classifying the cytoplasm of a motile cell and associated surface regions, into two types, lamelloplasm and endoplasm. In elongated unilamellar fibroblasts the lamelloplasm is anterior and the endoplasm is posterior, comprising the cytoplasm within the tail, often with a distinct boundary separating the two regions. After clearance from the lamelloplasmic surface, patches accumulate at this boundary, which, for reasons that will be discussed, we refer to as the 'null border.'

Because our goal was to use patches as markers of cell surface transport, it was necessary to determine if patch clearance is accompanied by patch internalization. Patches at the cell surface are accessible to antibodies applied briefly in the surrounding medium while internalized patches are not accessible. We used rhodaminelabeled rabbit anti-rat IgG to induce patch formation and then traced this rhodamine secondary antibody at various time points on living and fixed cells using a fluoresceinlabeled goat anti-rabbit IgG. There was little difference between the rhodamine and fluorescein distributions, indicating that the majority of patches were not internalized but remained at the cell surface during the $40 \mathrm{~min}$ in which capping occurred (data not shown). This result was typical for the majority of cells examined up to two hours after capping and demonstrated that only a small percentage of patches were internalized during clearance of patches from the lamella. By tracking movements of a relatively large number of patches per cell $(>25)$, we could be sure that almost all of the fluorescent structures were present in the plasma membrane rather than located in the cytoplasm in endocytic vesicles. For clarity, the tracking results below are illustrated using trajectories selected as representative of the sampled patch population.

Patches anterior and posterior to the boundary respond differently to retraction of the trailing edge: occurrence of a null border

We noted that forward movement of patches occurred in the posterior surface region when the cell shown in Fig. 2 underwent tail retraction. We then examined the simultaneous response of patches located anterior and posterior to the lamelloplasm/endoplasm boundary during spontaneous retraction of the trailing edge. Fig. 3 shows a cell $10 \mathrm{~min}$ after patch formation. Individual patch behavior was recorded by collecting sequential high-magnification fluorescence images of the inset area at approximately three minute intervals. A phase-contrast image and fluorescence images at two focal planes were acquired at each time point (see Materials and methods).

The movements of individual patches during the process of redistribution were tracked at both focal planes. Patch movements occurred relative to the substratum and are described as being rearward or forward on the basis of the perceived direction of cell movement. During the period of observation patches in focus at the higher focal plane moved continually forward at $0.2-0.5 \mu \mathrm{m} \mathrm{min} \mathrm{m}^{-1}$ toward the lamelloplasm/endoplasm boundary (Fig. 3D, white lines and arrow) but did not actually accumulate on the boundary. In contrast to this forward movement, some patches in focus at the lower focal plane moved continually rearward (Fig. 3D, black lines and arrow) also at velocities in the range of $0.2-0.5 \mu \mathrm{m} \mathrm{min} \mathrm{m}^{-1}$. Several of these patches eventually came into focus at the higher focal plane and so 

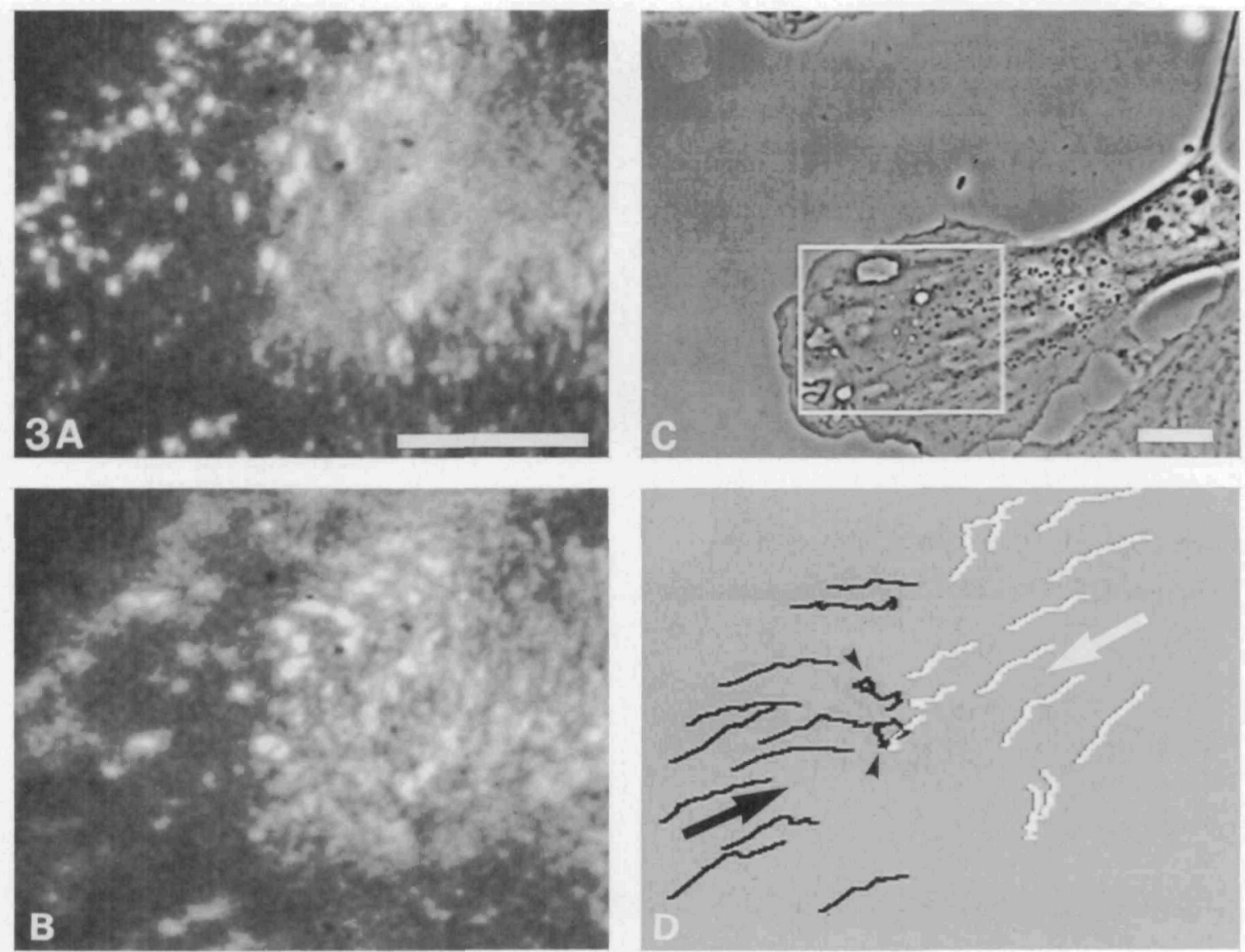

Fig. 3. Regionalized response of patches to retraction at the trailing edge. Movements of fluorescent patches were tracked from the series of lower (A) and higher (B) focal plane images. Fluorescence images were acquired from the region of the cell in $\mathrm{C}$ that is outlined in white. As the posterior portion of the cell underwent spontaneous retraction, more posterior patches shifted forward (white lines and arrow in D), while patches located closer to the leading edge continued to be transported rearward (black lines and arrow in D). These opposing patch movements converged at the approximate center of the imaged area. Two patches initially located at the site of convergence (black arrowheads in D) shifted slightly forward and rearward, but showed little net displacement. Both forward and rearward movements occurred on the dorsal cell surface (see text). Bar, 10 $\mu \mathrm{m}$.

we can conclude that these were located on the dorsal cell surface. Such patches will eventually accumulate on the boundary as they are cleared off the dorsal lamellar surface in the capping phenomena. Other patches in focus at the lower focal plane did not undergo significant rearward transport; these were most likely located on the ventral cell surface and did not move rearward due to obstruction by cell-substratum contacts. As a general trend, patches located on broad regions of the ventral surface were commonly found not to be cleared (for example, see Fig. 7, below). The opposed patch movements were not intermingled across the cell surface but occurred in distinct anterior and posterior regions, which were separated by an apparent boundary toward which the movements progressed. Two patches initially located close to this boundary (black arrowheads, Fig. 3D) shifted slightly forward and then rearward but underwent little net displacement.

The boundary phenomenon is also illustrated by patch movements in Fig. 4. Patches converged at a site just anterior to the nucleus during spontaneous retraction of the trailing edge. Note two features of the forward patch movements. First, displacements of individual patches correlated generally but not precisely with displacements of the nucleus and perinuclear organelles. For example, the nucleus moved little in the first four time intervals but then showed a surge forward. However, patches were already shifting forward during the early intervals; their movements reflected the forward surge of the nucleus.

Second, the trend of the more posterior patches to move a greater distance forward than more anterior patches is similar to the movements of posterior cytoplasmic organelles in response to trailing edge retraction described by Chen $(1981 a)$. Chen called the position where cytoplasmic vesicles ceased forward movement the 'null border.' Since the movements are similar, it seems reasonable to suggest that the boundary toward which Pgp-1 patches converge corresponds to the null border.

The distributions of patches on 25 of more than 50 cells examined were sufficiently clear and complete to allow us to confirm whether the null border phenomenon occurred. Patches converged toward a site located anterior to or over the nucleus on 23 of these cells and it was possible to track individual patches on both sides of the null border in seven of these. In the two other cells, rearward movement of patches persisted posterior to the nucleus. All these cells had a distinct protrusive leading lamella and nonprotrusive posterior cell margins.

The examples presented above illustrated patch movements occurring on cells that underwent little or no net 
migration. Translocation of a cell body is brought about by a coupling of tail retraction and extension of the leading edge in a cyclic process (Dunn, 1980). In the preceding examples we followed patch movements only during the initial portion of this cycle. The behavior of the null border and endoplasmic patches on a cell undergoing net migration is illustrated in Fig. 5. Following clearance of patches from the dorsal lamellar surface we photobleached lines perpendicular to the elongated cell body at various distances from the boundary. Subsequent to photobleaching, fluorescence and phase-contrast images were collected and movements of the photobleached lines were followed. Comparison of Fig. 5A and E shows that the anterior cell margin advanced approximately $10 \mu \mathrm{m}$ and the lamella became broader. The posterior portion of the cell became more narrow as the nucleus and perinuclear organelles shifted forward, but the tail did not become completely detached from the substratum. As indicated by deformation and movement of the parallel bleached lines, a forward shift of patches (Fig. 5B-D) accompanied the forward shift of the endoplasmic cytoplasm (Fig. 5E). The null border (Fig. 5B-D, broken lines) advanced with migration of the cell and the contour of the null border became broader, accommodating the change in shape of the lamella. Note that patches have been transported forward with respect to both the substratum and the forward moving null border. We believe that the bleached lines are not 'sharp' because of the difficulty of keeping the thicker tail in focus, as opposed to the lines being eroded by lateral diffusion of the patches (see below).

As a control, we attempted to record behavior of individual patches on the endoplasmic surface in the absence of posterior retraction. However, each polarized cell examined showed some posterior retraction, and consequently in each case endoplasmic patches showed a shift forward. Therefore, we turned to a subpopulation of especially large, often binucleate cells that are well spread and commonly clear patches only from a peripheral zone $10 \mu \mathrm{m}$ wide. When a line pattern was photobleached in the central surface region after patching Pgp-1, the pattern remained distinct for more than 30 min (data not shown), indicating that the surrounding patches were very limited in their ability to undergo long-range diffusion. Because these cells in the region bleached were flat and thin, the bleached marks were in focus over their entire extent and remained so for the duration of the experiment. This conclusion is consistent with recent fluorescence recovery after photobleaching measurements on patched Pgp-1 (Holifield et al. 1990).

In examples shown above, forward and rearward patch movements occurred parallel to the axis of tail retraction and converged at the null border. A more complex situation arose when patches on the lateral aspect of a leading lamella moved rearward and approached the null border at an angle to the axis of retraction. Fig. 6 illustrates the results. Patches located on the dorsal endoplasmic surface moved continuously forward along the long axis of this cell (left arrow, in A). When patches moving rearward (right arrow, in A), eventually approached the null border, rather than stopping and accumulating there, they turned approximately $90 \mathrm{de}$ grees and began to move forward in the vicinity of the null border and along the long axis of the retracting cell body in concert with the forward-moving patches on the endoplasmic surface. In $\mathrm{F}$ compare the bent broken white arrow depicting the turning behavior of initially rearwardmoving patches with the filled straight white arrow depicting the forward-moving endoplasmic patches. In this panel, the approximate location of the null border is depicted by the thin black line.

\section{Regional patch behavior shows correlation with the} organization of $F$-actin

Since rearward transport is confined to a distinct cell surface region, and since contraction within a cortical actin meshwork is implicated in driving this transport (Dunn, 1980; Heath, 1983b), we examined whether F-actin shows a regionalized organization related to the behavior of patches. The cell shown in Fig. 7 was fixed 40 min after patch formation and then stained with rhodaminephalloidin to visualize F-actin. Three focal planes are shown. Note that patches remain in the lamellar region at the ventral focal plane (Fig. 7A). At a higher plane of focus, patches on the dorsal surface are seen to have accumulated in the vicinity of the expected lamelloplasm/ endoplasm boundary (Fig. 7A and B). Patches in focus at the cell apex behind the boundary have not coalesced into more dense aggregates but retain their individuality (Fig. 7C). The F-actin organization at corresponding focal planes is shown in the Fig. 7D-F. Note the fine fibrillar arrangement of F-actin that came into sharp focus as the focal plane was raised above the substratum (Fig. 7E). On the basis of the focus at different levels, this assembly of F-actin can be recognized to underlie the dorsal plasma membrane. Also note that the posterior edge of this dorsal cortical actin sheath was distinct at the highest focal plane (arrow, Fig. 7F) and that the location of this posterior edge corresponded well to the site where patches accumulated (arrow, Fig. 7C). There was no prominent network of F-actin underlying the surface posterior to the site of patch accumulation. The posterior edge of the dorsal F-actin sheath and the site of patch accumulation commonly coincided when the edge of the sheath was detectable amidst the background of out-of-focus structure.

The dorsal cortical F-actin sheath in fibroblasts gives rise to characteristic F-actin bundles, called arcs, oriented approximately parallel to the leading cell margin (Heath, 1981, 1983a; Sorrano and Bell, 1982). When arcs are present during rearward transport of adsorbed particles (Heath, 1983a) or membrane protein patches (Heath, $1983 b$ ), these surface markers move in concert with the arcs. We corroborated Heath's finding that patches accumulate over and move rearward in concert with moving arcs. Fig. 8 shows a sequence of phase-contrast and fluorescence images illustrating this. Note that as a phase-dense arc forms and moves rearward (arrows, in A and B), patches become more dense immediately over it and move in concert. This cell was fixed and stained with NBD-phallacidin to confirm that the phase-dense structure was an $\mathrm{F}$-actin bundle (arrow, in $\mathrm{C}$ ).

\section{Discussion}

Lateral patch transport as a reflection of membrane cortical cytoskeletal behavior during cell locomotion

Patch transport phenomena can be interpreted to reveal information concerning the behavior of the plasma membrane and the associated cortical cytoskeleton during cell locomotion. We postulate that plasma membrane patches of Pgp-1, large aggregates composed of hundreds of proteins (Jacobson et al. 1984), serve as markers for the cortical cytoskeleton. There is highly suggestive, although circumstantial, evidence for patches being linked to the 

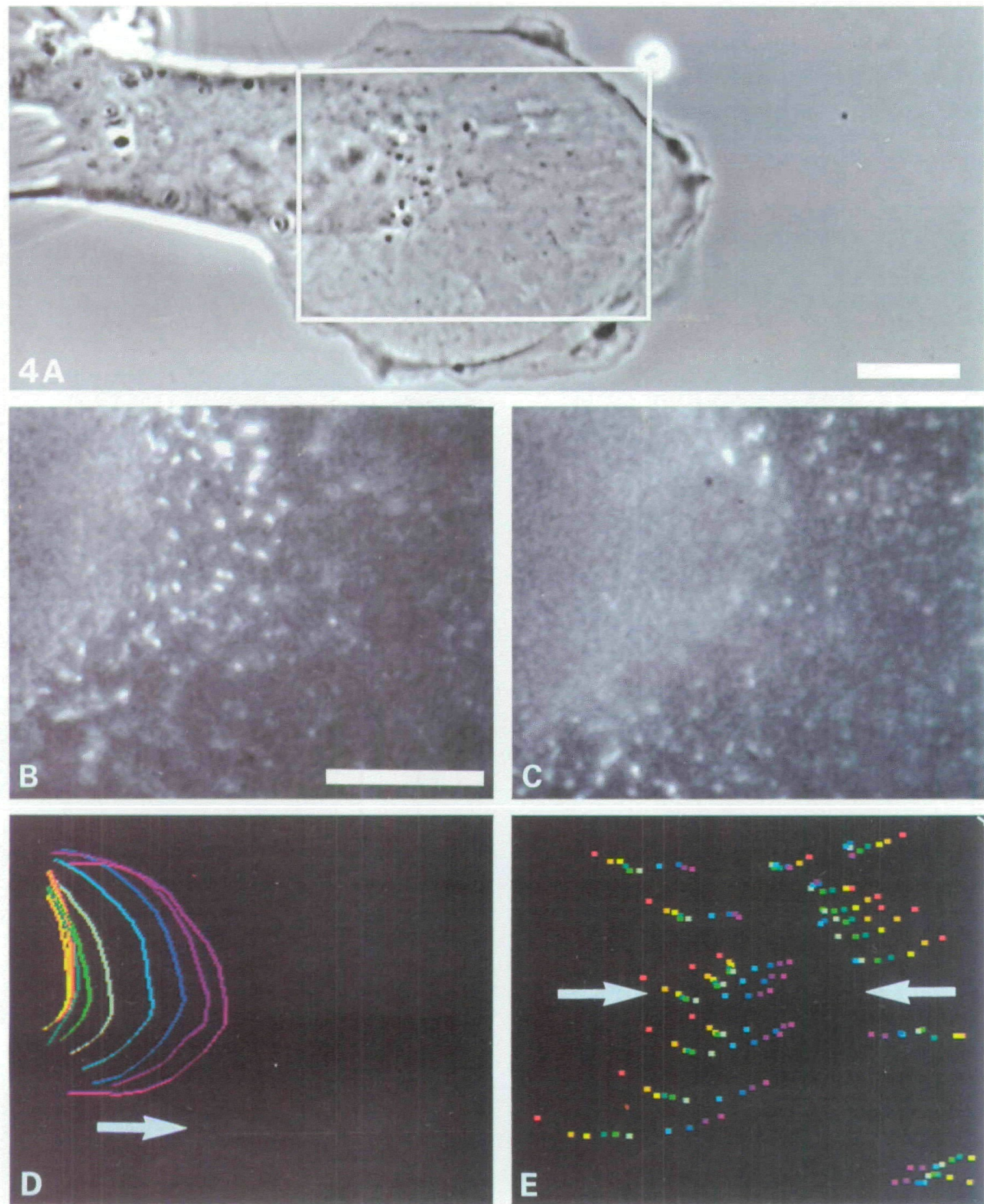

Fig. 4. Forward movement of patches during posterior retraction correlates generally with forward movement of the nucleus. The cell in $A$ is shown after acquiring a series of fluorescence images from a higher (B) and a lower focal plane (C). The boxed area in $A$ is the region in which patches were tracked. Sequential positions of the anterior edge of the nucleus (D) and patches (E) are color coded, starting with red and progressing to violet. Time points span approximately 3 -min intervals. Bars, $10 \mu \mathrm{m}$. 


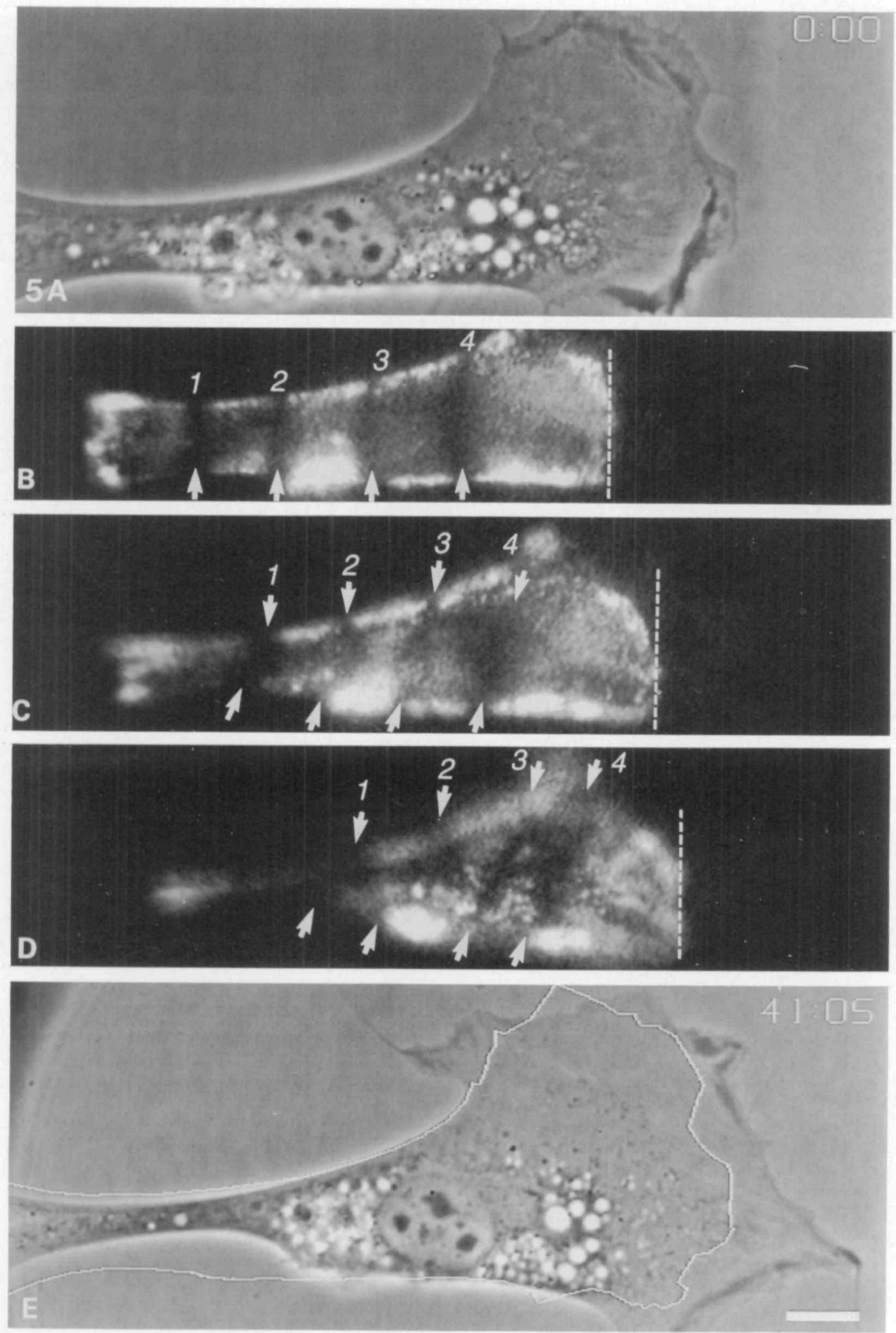

Fig. 5. Forward movement of the null border accompanies translocation of the cell body. This cell was observed for $40 \mathrm{~min}$, during which time the leading edge advanced over $10 \mu \mathrm{m}$ as the tail progressively narrowed (compare A and E). Forward movement of patches accumulated at the null border (broken lines in B-D) accompanied migration of the cell. Four parallel lines were bleached across the elongated posterior cell body (arrows in B-D). As the tail narrowed, these lines were deformed and shifted forward. Note that progressive narrowing of the tail was accompanied by progressive forward redistribution of patches, and that this occurred prior to tail detachment. Changing cell thickness during retraction makes some defocusing unavoidable. White outline in $\mathrm{E}$ indicates original position of cell taken from A. Bar, $10 \mu \mathrm{m}$. 

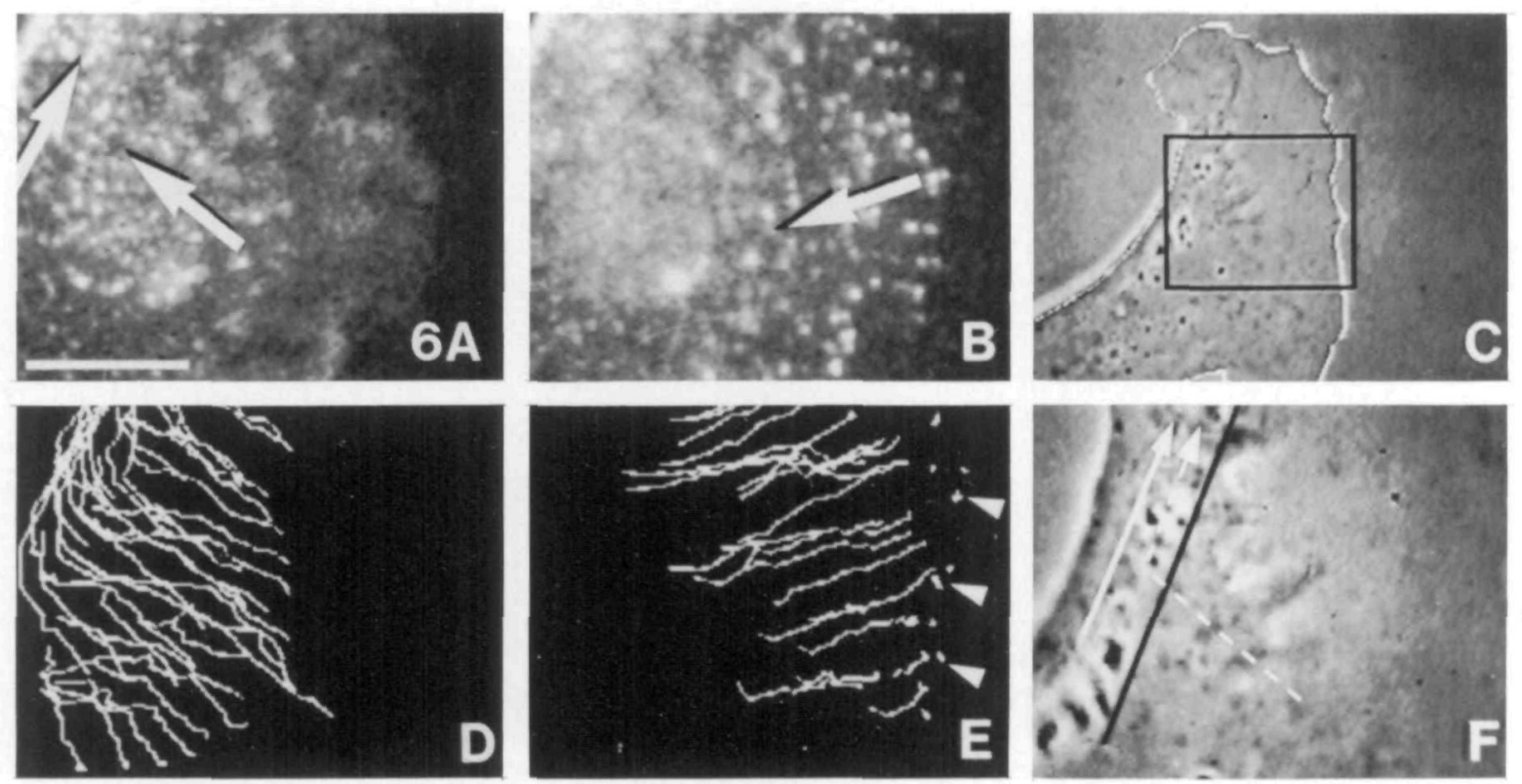

Fig. 6. Turning behavior of patches approaching the null border. Fluorescent patches were imaged at higher (A) and lower (B) focal planes in the boxed area of the cell shown in phase-contrast in $\mathrm{C}$. The boxed area is shown at higher magnification in $\mathrm{F}$ corresponding to the fluorescent images in $\mathrm{A}$ and $\mathrm{B}$. The axes of rearward and forward patch movements within the boxed area in $\mathrm{C}$ were not parallel in this cell. Consequently, non-convergent movement of lamellar and endoplasmic patches occurred. As dorsal patches moved rearward and approached the vicinity of the null border (identified approximately by the black line in F), they turned approximately 90 degrees and joined in the ongoing forward patch movement occurring behind the null border. This is depicted by the trajectories in $\mathrm{D}$ and the arrows in $\mathrm{A}$. The phase-contrast morphology of the null border area, and the directions of dorsal patch movements are shown in $\mathrm{F}$, where the bent, broken white arrow indicates the turning behavior of patches approaching the null border from the right on the lamellar surface while the filled white arrow indicates the direction of patch movements on the endoplasmic surface behind the null border. On the ventral cell surface, some patches were not transported rearward (arrowheads in E), while others moved away from the cell margin (E) but at an angle different from that moved by overlying patches on the dorsal surface (compare arrows in A and B). Bar, $10 \mu \mathrm{m}$.

membrane-associated cortical cytoskeleton. Crosslinked antigens, in general, align either with or between microfilament bundles (Singer et al. 1978; Jacobson et al. 1984). As described above, bleached marks on Pgp-1 patch arrows in highly spread, largely stationary cells showed no evidence of diffusional erosion over periods in excess of $30 \mathrm{~min}$; this observation indicates that the patches are immobilized, presumably by anchorage to the cortical cytoskeleton. Indeed, in lymphoid cells crosslinked surface immunoglobulins (Braun et al. 1982; Woda and McFadden, 1983; Salisbury et al. 1988) as well as other antigens (Turner and Shotton, 1989) remain with the detergentinsoluble cytoskeleton. Finally, the mechanism of capping seems largely resolved in terms of coupling of rearward patch transport to a retrograde cortical actin flow (see below). Thus we can provisionally use the movements of patches to produce inferences about the dynamics of the subjacent membrane cortex in motile cells.

\section{Capping: regionalized rearward transport of patches on the lamellar surface}

Various mechanisms have been proposed to explain the rearward transport of protein patches, such as seen for Pgp-1. These are based on two general themes: either patches are transported by a rearward flow of membrane components caused by coupling a source of membrane at the leading edge to a sink distributed over the rest of the cell (Abercrombie et al. 1972; Bretscher, 1976, 1984; Harris, 1976), or patches move rearward as a consequence of coupling to a constitutive rearward flow of cortical cytoskeletal components (Dunn, 1980; Abercrombie, 1980;
Dembo and Harris, 1981; Heath, 1983b; Bray and White, 1988). Evidence supporting each of these general mechanisms for capping has generated much debate.

Insertion of membrane proteins at the leading edge is supported by direct evidence (Marcus, 1962; Bergmann et al. 1983). However, the idea that such insertion drives a flow of proteins or lipids away from the leading edge is based on indirect evidence (Bretscher, 1984) and there has been no demonstration of a large forward flux of vesicular material through the lamelloplasm to sustain large-scale membrane insertion at the leading edge (Fisher et al. 1988; Forscher and Smith, 1990, and references therein).

The preponderance of recent evidence now clearly favors a cytoskeletally based mechanism that utilizes a retrograde cortical actin flow to clear patches from the leading lamella. The ultrastructural organization of cortical microfilaments has been described (Heath, 1981, 1983a). There is strong evidence that elements of this actin-based cortex undergo a prominent rearward flux in motile cells (Sorrano and Bell, 1982; Heath, 1983b; Wang, 1985; Forscher and Smith, 1988; Fisher et al. 1988). Furthermore, patches and inert particles adsorbed to the cell surface are transported rearward in concert with the observable flow of actin-based structures (Heath, 1983a,b; Fisher et al. 1988; Forscher and Smith, 1990). Several direct tests have demonstrated that the alternate retrograde lipid-flow hypothesis of capping (Bretscher, 1976, 1984) is incorrect (Sheetz et al. 1989; Lee et al. 1990; Kucik et al. 1990; Holifield et al. 1990). All of these recent studies point to the alternative mechanism in which aggregated components are selectively transported through the plane 

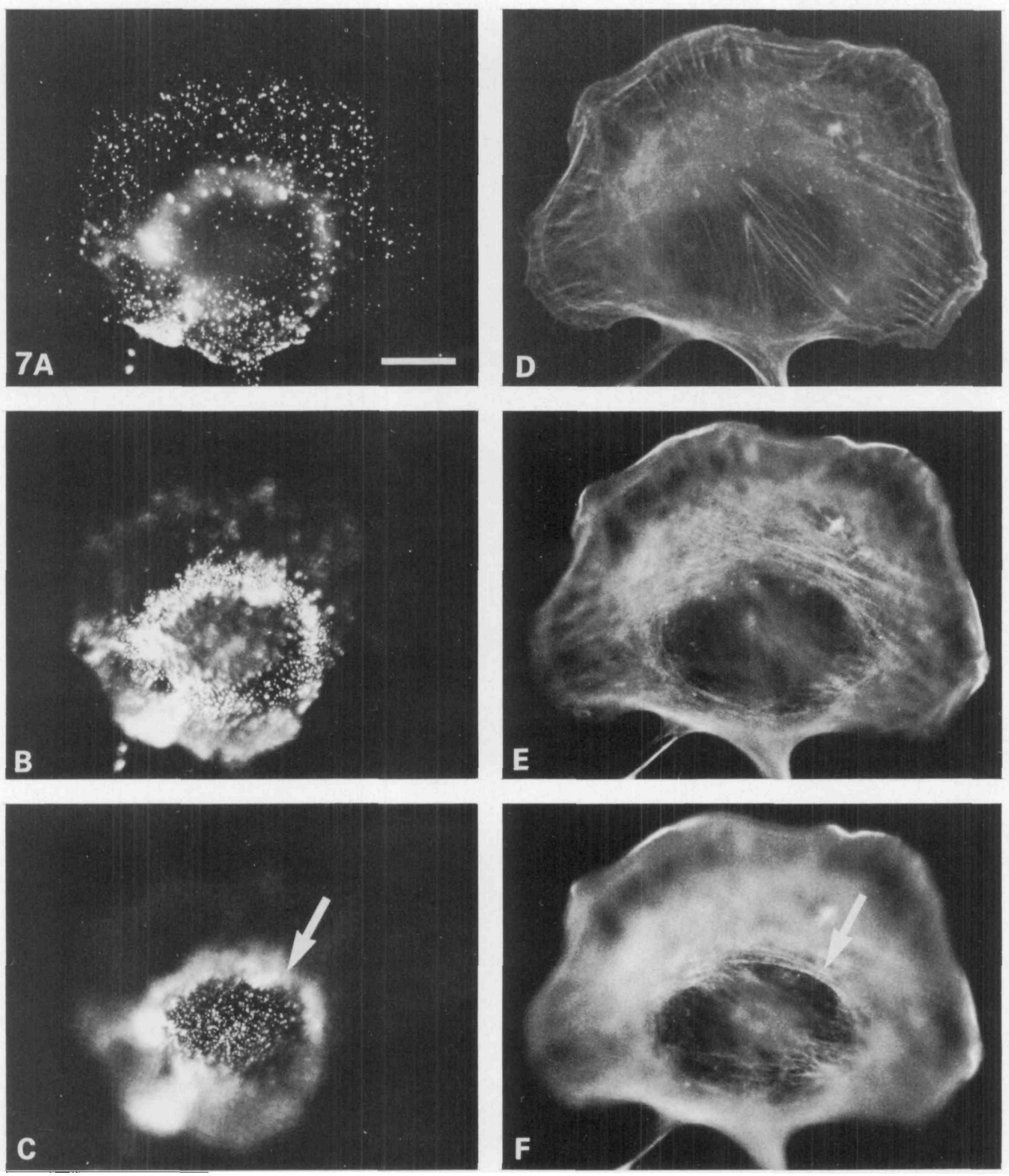

Fig. 7. The site of patch accumulation corresponds to the posterior edge of the dorsal cortical F-actin sheath. Pgp-1 patches are shown at three focal planes in A-C, the lowest (A) corresponding to the ventral cell surface and the highest plane (C) is at the cell apex. The distribution of $\mathrm{F}$-actin labelled with rhodamine-phalloidin is shown at the corresponding focal planes in D-F. Patches were not cleared from a broad portion of the ventral surface (A), but were cleared from the dorsal surface and accumulated at the typical surface location (arrow in C). As the focus level rose above the substratum, a dense arrangement of F-actin came into focus ( $\mathrm{E}$ and F). This dorsal cortical F-actin sheath showed a discrete posterior edge that corresponds to the site of patch accumulation (compare arrows in $\mathrm{C}$ and $\mathrm{F}$ ). Bar, $10 \mu \mathrm{m}$. 

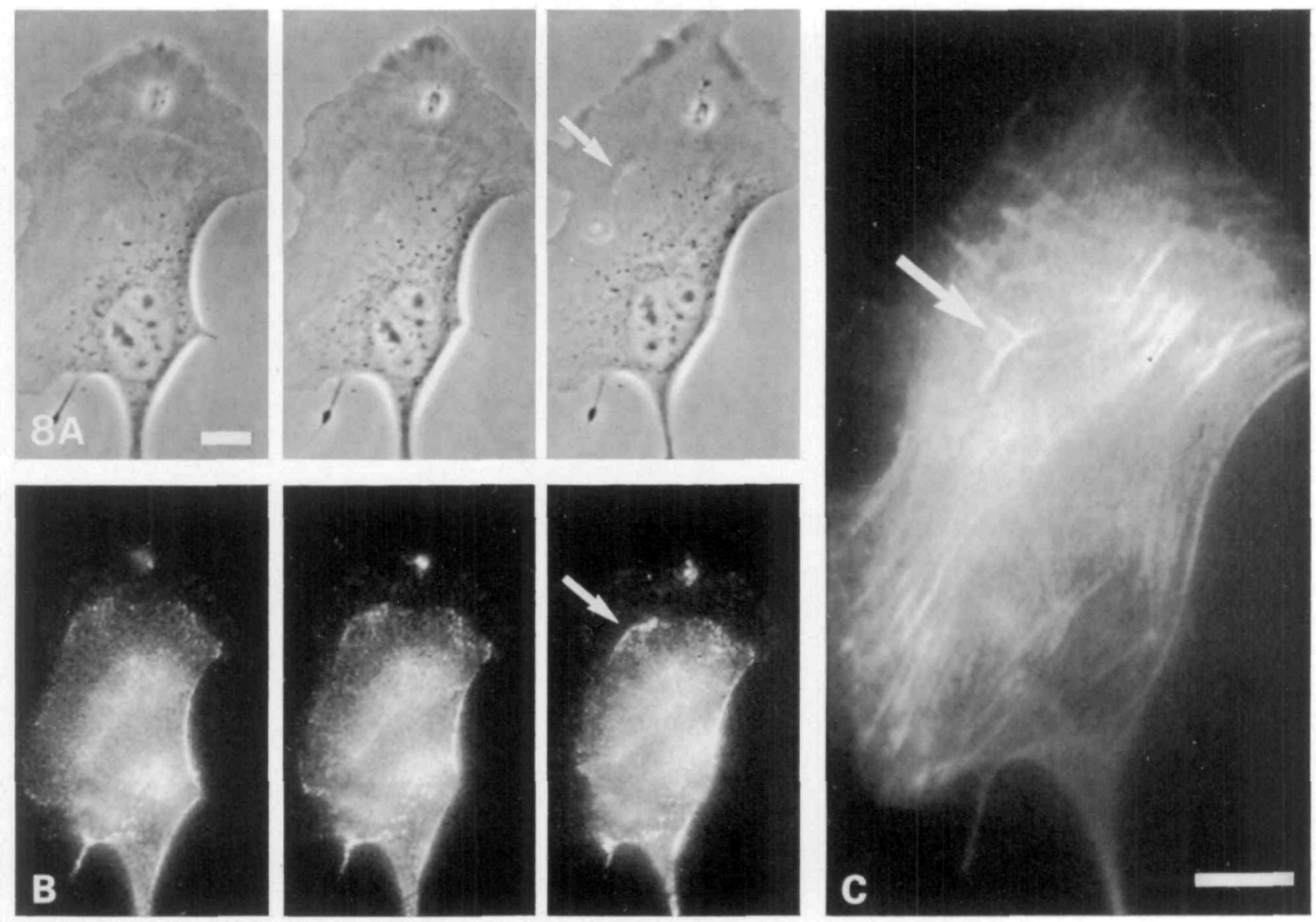

Fig. 8. Patches accumulate over and move rearward with F-actin arcs in C3H 10T1/2 fibroblasts. A sequence of phase-contrast images spanning $4 \mathrm{~min}$ is shown in $\mathrm{A}$, and the corresponding patch distributions are shown in $\mathrm{B}$. During this time period, as a phase-dense arc formed and moved inward, patches on the dorsal surface become more concentrated directly over the arc (arrows in $A$ and B). The cell was fixed and stained with NBD-phallacidin to visualize F-actin (C). The phase-dense arc in A corresponds to a curved $\mathrm{F}$-actin bundle (compare arrows in $\mathrm{A}$ and $\mathrm{C}$ ). Bars, $10 \mu \mathrm{m}$.

of the plasma membrane by a contractile cortical process without an accompanying rearward flow of lipid and nonaggregated membrane components.

The force-producing mechanism responsible for both the retrograde cortical actin flow and capping is considered to be at least in part based on an actomyosin cortical contraction (Bray and White, 1988). This is supported by recent evidence that myosin-deficient Dictyostelium mutants are unable to cap (Pasternak et al. 1989). The retrograde cortical actin flow also may be sustained by actin treadmilling (Wang, 1985) or by an appropriate disposition of myosin I type motors (Fukui et al. 1989; for reviews, see Smith, 1988; Mitchison and Kirschner, 1988; Adams and Pollard, 1989).

The finding that patches accumulate over and move rearward in concert with curved $\mathrm{F}$-actin-containing arcs during capping (Fig. 8) is consistent with the cortical cytoskeletal flux hypothesis. Arcs are especially dense regions of the dorsal cortical microfilament sheath located in the leading lamella. This study extends the work of Heath $(1983 b)$ in that our observations were made using a different cell type and different plasma membrane protein, indicating that the relationship between arcs and patched antigens is more widespread than previously discussed. Furthermore, the present study shows that patches are transported rearward only over the cell surface region overlying the dorsal cortical F-actin sheath (Fig.7).
Patches accumulated densely at the posterior edge of this sheath, usually located just anterior to the nucleus. Posterior to this site, patches appeared to remain randomly dispersed and the actin cortex is not organized into arc-shaped bundles.

It has long been recognized that patches on fibroblasts accumulate in front of the nucleus after capping, but the relationship between the site of patch accumulation and the regional organization of F-actin has not been shown previously, although the observation that arcs fade from view near this site (Heath, 1983a,b) suggests that there is such a relationship. Early studies on capping suggested that the rearward flow was directed toward sinks for membrane internalization. However, one result from the present study argues against a sink being responsible for drawing patches rearward to this site. In Fig. 6, patches on either side of the null border moved at almost right angles to one another. If a discrete sink for membrane uptake were drawing patches located on the lamella rearward, then the slightly more posterior patches on the endoplasmic surface should also have been drawn directly to the sink as well. Instead, endoplasmic patches located 3-5 $\mu \mathrm{m}$ behind the null border, which was recognizable by phasecontrast, moved parallel to it. Also, if this null border were the site of a sink, rearward-moving patches should have stopped upon reaching the sink, but instead they turned approximately 90 degrees and joined the endoplasmic 
patches moving, as a result of tail retraction, anteriorly down the long axis of the cell. These studies do not preclude the possibility of the null border being a site for membrane uptake, but suggest that if endocytosis does occur there it does not induce large-scale membrane flow toward this site.

\section{Cell surface dynamics during retraction: forward patch transport on the tail surface}

The process of tail retraction has been shown to consist of ATP-independent and ATP-dependent components (Chen, $1981 a$ ), suggesting that the mechanism of tail retraction involves both the visco-elastic mechanical properties of the cortical microfilament network and a contractile activitiy inherent in this actomyosin-based network. Contractile stress fibers in a motile fibroblast are commonly observed to run the length of the tail and to merge into a looser network as they approach the perinuclear region. However, contraction of stress fibers in the tail leads to forward movement of both the surface and cytoplasm of the tail region, rather than clearance of patches from the surface of the tail (see Fig. 5), consistent with Chen's observations. We make the inference from the forward patch transport that the dorsal cortical cytoskeleton in this region of the cell also shifts forward. The fact that displacements per unit time are generally larger at more distal positions from the null border may be due to either of two factors. First, these graded displacements could be due to an elastic recoil of the cortical cytoskeleton anchored at the null border coupled with a folding or blebbing of the bilayer between patches. (The bilayer itself will not sustain a large compression and thus must fold upon retraction.) Alternatively, near the null border, dorsal folding of the membrane (Chen, 1981a) and the subjacent cortex could, in two-dimensional projection, give rise to smaller displacements near the null border.

\section{Forward patch transport occurs only behind the null border}

Forward movement of aggregated integral plasma membrane components in response to retraction of the posterior cell body in itself was not an unexpected result, but two aspects of this forward shift were not intuitive. The first was that the observed response of the cell surface to posterior retraction was discontinuous along the length of the cell. The entire cell body of an elongated cell appears to be under tension, as shown by its tendency to retract at either the anterior or posterior end when substratum adhesions are broken, for example, by trypsinization. For this reason, a forward shift of patches in response to tail retraction might be expected to be distributed along the entire length of a cell. In fact, the forward shift of patches was confined to the endoplasmic surface region, behind the null border. This discontinuity reveals the striking regionalization of cortical cytoskeletal behavior.

It has been suggested that patches of aggregated membrane proteins interact with the cortical cytoskeleton by means of a subset of integral membrane proteins (Bourguignon and Singer, 1977; Singer et al. 1978). These molecules would act like the tines of a rake and constrain patches to move in concert with the cortical cytoskeleton, as previously proposed (de Petris, 1978; Dunn, 1980; Dembo and Harris, 1981; Holifield et al. 1990). Because the rearward flux of the cortical sheath in the lamellar region appears to cease at the null border, the suggested raking effect would cease as well, perhaps due to disassembly of cortical cytoskeletal components or to some other means of uncoupling the tines from the rake. As the rearward raking action ceases at the null border, the forward recoil of the tail during retraction would provide the impetus for patches behind the null border to shift forward. These patches would be constrained to remain at or behind the null border, since any patches that might 'escape' beyond the null border would be quickly swept back by the suggested raking action. Thus, confinement of the rearward raking action to the region anterior to the null border would be responsible for the observed discontinuity in response of patches to tail retraction. Over the short term, patches situated directly on the null border would show little net directed movement because at that site the rearward- and forward-directed forces acting on them would be balanced. Over the long term, patches would move forward beyond the initial site of the null border only when the cortical components of the endoplasm shift forward upon translocation of the cell body (Fig. 5). It is important to realize that while forward movement of patches ceases at the null border because of the interplay of regionalized cortical cytoskeletal behavior, forward movement of the lipid bilayer and non-aggregated proteins is probably not restrained. Presuming that the membrane reservoir for leading edge extension is provided by trailing edge retraction, the bilayer must be permitted to flow forward across the null border (Ishihara et al. 1988; Lee et al. 1990).

Forward patch transport occurs continuously during the elongation and narrowing phase preceding overt detachment

The second novel finding was that forward movement of the posterior cell surface, as indicated by the movement of patches, appeared to occur continuously prior to tail detachment, during elongation and narrowing of the cell body during cell migration. Progressive narrowing and elongation of the cell body is commonly observed in populations of migratory fibroblasts (Abercrombie et al. 1977). Cells appear to be 'stretched' by a continual advance of the leading cell margin while the posterior tip remains stationary. This stretching appears to generate tension and frequently leads to abrupt detachment of the tail (Chen, 1979). Results shown in Fig. 5 indicate that a forward movement of aggregated cell surface components and, by inference the subjacent cortex accompanies not only tail detachment and its forward recoil, but also the gradual elongation and narrowing that precedes tail detachment. This more gradual forward redistribution of the. cell surface may indicate that cell-substratum adhesions yield to the increasing tension sequentially according to their size or strength, for example close contacts and then focal contacts, or that some elements maintaining the tension do not extend all the way to the tip of the tail. Since this gradual forward surface redistribution is a common feature of cell locomotion, an accurate view of the behavior of the cell surface during locomotion must take it into account.

\section{Conclusion}

Locomotion of cells in culture can be viewed as a continual cycle of spreading, traction and tension development, followed by trailing edge retraction. By assuming that aggregates of Pgp-1 located in the plasma membrane serve as markers for the subjacent membrane-associated cytoskeletal cortex, we can gain insight into the cortical 
aspects of cell surface dynamics during locomotion. At this juncture, the relationship of rearward patch transport during capping to the mechanism of leading edge extension is not clear. However, the retrograde capping of lamellar patches may well be related to retraction in that the driving force for the retrograde cortical actin flow, when coupled to stationary ventral surface-substratum contacts, may demand that the tail be pulled forward. It is important to note that this active region of the cortex, as reflected by rearward patch transport, appears strictly confined to regions ahead of the null border. Furthermore, retraction, as reflected by anterior patch movements on the cell surface, is also highly regionalized and separated from the dynamic lamellar region as judged by the failure of patches on the endoplasmic surface to proceed forward beyond the null border. During retraction, forward patch transport, with respect to both the substratum and the null border, occurs during the elongation and narrowing phase of the distal portion of the trailing edge; it is generally coupled to forward organelle transport, though not in perfect register. Confinement of forward patch transport to regions behind the null border probably does not apply to the forward movements of the bilayer itself. Such bulk forward movement of the bilayer, which may be required to provide membrane for leading edge extension, would be expected to be continuous across the null border. We can expect that additional tracing of the movements of defined membrane and cortical components will further elucidate the dynamics of the cell surface in moving cells.

This work was supported by NIH grant GM35325 and ACS grant CD-181. We thank Mr You Hou for technical assistance, and Dr James DiGusseppi and Dr Richard Inman for writing some of the image analysis software used in this study. We also acknowledge the numerous helpful discussions with Dr Keith Burridge during this project and thank Dr Greta Lee for her critical reading of this manuscript. We acknowledge the helpful comments of the referees.

\section{References}

Abercrombir, M. (1980) The crawling movement of metazoan cells. Proc. R. Sac. Lond. B 207, 129-147.

Agercromeie, M., Dunn, G. A and Hzath, J. P. (1977). The shape and movement of fibroblasts in culture. In Cell and Tissue Interactrons (ed. J. W. Lash and M. M. Burger), pp. 57-70. Raven Press, NY.

Abrrcrombie, M., Heaysman, J. E. M. and Pegrum, S. M. (1970a). The locomotion of fibroblasts in culture. II. 'Ruffling'. Expl Cell Res. 60, $437-444$.

Abercrombie, M., Heaysman, J. E. M. and Prgrum, S. M. (1970b). The locomotion of fibroblasts in culture. III. Movements of particles on the dorgal aurface of the leading lamella. Expl Cell Res 62, 389-398.

Aberchombie, M., Henysman, J. E. M. and Pegrum, S. M. (1972). Locomotion of fibroblasts in culture. V. Surface marking with concavalin A. Expl Cell Res, 73, 536-539.

Adams, R. J. AND Pollakd, T. D. (1989). Membrane-bound myosin-1 provides new mechanisms in cell motility. Cell Motil. Cytoskel. 14, 178-182.

Belitgog, P. C., Hildreth, J. and August, J. T. (1990). Homotypic cell aggregation induced by anti-CD44 ( $\mathrm{Pgp}-1$ ) monoclonal antibodies and related to CD44 (Pgp-1) expression J.Immun. 144, 1661-1670.

Brrganan, J. E., Kupfer, A and Singre, S. J. (1983). Membrane insertion at the leading edge of motile fibroblasts. Proc. natn. Acad. Scl. U S.A 80, 1367-1371.

Bourguignon, L. Y. and Singer, S. J (1977). Trangmembrane interactions and the mechanism of capping of surface receptors by their specific ligands. Proc. natn. Acad. Sci. U.S.A. 74, 5031-5035.

Braun, J., Hockman, P. S. and Unanue, E. R. (1982). Ligand-inducod agsociation of surface immunoglobulin with the detergent-insoluble cytoskeletal matrix of the B-lymphocyte. J Immun 128, 1198-1204.

Bray, D. AND White, J. G. (1988). Cortical flow in animal cells. Science 239, 883-888.
Bretscher, M. S. (1976). Directed lipid flow in cell membranes. Nature 260, 21-23.

BRETrCHFR, M. S. (1984). Endocytosis: relation to capping and cell locomotion. Science 224, 681-686.

CARTER, W. G. AND WAYNER, E. A. (1988). Characterization of the clase III collagen receptor, a phosphorylated, transmembrane glycoprotein expressed in nucleated human cells J. biol. Chem. 263, 4193.

CHEN, W.T. (1979). Induction of spreading during fibroblast movement. J. Cell Bıol. 81, 684-691.

Chen, W.-T. (1981a). Mechanım of retraction of the trailing edge during flbroblast movement J. Cell Biol. 90, 187-200

Chen, W.-T. (1981b). Surface changes during retraction-induced spreading of fibroblasts. J. Cell Sci. 49, 1-13.

Dembo, M. And Harris, A. K. (1981). Motion of particles adhering to the leading lamella of crawling cells. J. Cell Biol. 91, 528-536

DE Pktris, S (1978). Non-uniform distribution of Concanavalın-A receptors and surface antigens on uropod-forming thymocytes. $J$ Cell Biol. 79, 235-251.

DiGulgeppi, J., Inman, R., Ighihara, A., Jacobson, K. and Herman, B (1985). Applications of digitized fluorescence microscopy to problems in cell biology. Blotechniques 3, 394-403.

DunN, G. A. (1980). Mechanisms of fibroblast locomotions in cell adhesion and motility. The Third Symposumm of the Britzsh Saclety for Cell Biology (ed. A. S. G. Curtis and J. D. Pitts), pp. 409-423. Cambridge University Press, Cambridge.

EDIDIN, M. AND WEI8s, A. (1972). Antigen cap formation in cultured fibroblasts: a reflection of membrane fluidity and of cell motility Prac. natn. Acad Scl U.S.A. 69, 2456-2459.

Fisher, G. W., Conrad, P. A., DeBiasio, R. L. and Taylor, D. L. (1988), Centripetal transport of cytoplasm, actin, and the cell surface in lamellipodia of fibroblasts. Cell Motil Cytoskel. 11, 235-247

ForschrR, P. AND Smith, S. J. (1988). Actions of cytochalasins on the organization of actin filaments and microtubules in a neuronal growth cone J. Cell Biol. 107, 1505-1516.

ForschBr, P. AND Smith, S. J. (1990). Cytoplasmic actin filaments move particles on the surface of a neuronal growth cone. In Optical Microscopy for Biology (ed. B. Herman and K. Jacobson), pp. 459-471. Wiley-Liss, New York.

Fukui, Y., Lynch, T. J., Brzeska, H. and Korn, E. D (1989). Myosin 1 is located at the leading edge of locomoting Dictyostelium amoeba. Nature 341, 328-331.

HarRis, A. AND DUNn, G. (1972). Centripetal transport of attached particles on both surfaces of moving fibroblasts. Expl Cell Res. 73 519-523.

HARRIs, A. K. (1973). Cell surface movements related to cell locomotion. Locomotion of Tussue Cells. A CIBA Foundation Sympostum.

Harris, A. K. (1976). Recycling of disgolved plasma membrane components as an explanation of the capping phenomenon. Nature 263, 781-783.

Heath, J. P. (1981). Arcs: curved microfilament bundles beneath the dorsal surface of the leading lamellae of moving chick embryo fibroblasts. Cell Biol. Int. Rep. 5, 975-980.

Heath, J. P. (1983a). Behaviour and structure of the leading lamella in moving fibroblasts. I. Occurrence and centripetal movement of arcshaped microfilament bundles beneath the dorsal cell surface. $J$. Cell Sci. 60, 331-354

Heath, J. P. (1983b). Direct evidence for microfilament-mediated capping of surface receptors on crawling fibroblasts. Nature $\mathbf{3 0 2}$ $532-534$

Holifield, B., Ishihara, A. AND Jacorgon, K. (1990). Comparative behavior of membrane protein-antibody complexes on motile fibroblasts: Implications for a mechanism of capping. J. Cell Biol. 111, 2499-2512.

Hughes, E. N. AND AugusT, J. T. (1981). Characterization of plasma membrane proteins identified by monoclonal antibodies. J. biol. Chem. 256, 664-671

Ishihara, A., Holifield, B. AND JaCOBson, K. (1988). An analybis of lateral redistribution of a plasma membrane glycoprotein-monoclonal antibody complex which occurs during cell locomotion. J. Cell Biol $106,329-343$

Jacobson, K., O'Dell, D., Holifield, B., Murphy, T. L and August, J. T. (1984). Redistribution of a major cell surface glycoprotein during cell movement. J. Cell Biol. 99, 1613-1623.

Koppel, D. E., Oliver, J M. and Berlin, R. D. (1982). Surface functions during mitosis. III. Quantitative analysis of ligand-receptor movement into the cleavage furrow: diffugion v8. flow. J. Cell Btol. 93 $950-960$

KuciK, D. F., Elson, E. L. And Shegtz, M. D. (1990). Cell migration does not produce membrane flow. J. Cell Biol. 111, 1617-1622.

Lez, J., Gugtargeon, M , Magnusgon, K.-E. and Jacobson, K. (1990). The direction of lipid flow in locomoting polymorphonuclear leukocytes. Science 247, 1229-1233. 
Marcus, P. I. (1962). Dymamics of surface modification in myxovirusinfected cells. Cold Spring Harbor Symp. quant. Biol. 27, 351-365. Mitchison, T. AND Kirschner, M. (1988). Cytaskeletal dynamics and nerve growth. Neuron 1, 761-772.

Pasternak, C., Spudich, J. A. and Elson, E. L. (1989). Capping of surface receptors and concomitant cortical tension are generated by conventional myosin. Nature 341, 649-651.

SalibBury, J. L., Baron, A. T., Keller, G. A. ANd Skiest, D. (1988). Membrane IgM: Interactions with the cortical cytoskeleton in the human lymphoblastoid cell line WiL2. Cell Motil. Cytoskel $\boldsymbol{\theta}$, $140-152$.

Sheetz, M. D., Turney, S, Qian, H. and Elson, E L. (1989),

Nanometre-level analysis demonstrates that lipid flow does not drive membrane glycoprotein motions. Nature 340, 284-288.

Singer, S. J., Ash, J. F., Bourauignon, L. Y. W., Hegannegs, M. H. ANBD LoUVA RD, D. (1978). Transmembrane interactions and the mechenisms of transport of proteins across membranes. $J$. supramolec. Structure 9, 373-389.

SMITH, S. J. (1988). Neuronal cytomechanics: the actin-based motility of growth cones. Scrence 242, 708-715.

Soranno, T. AND BeL, E. (1982). Cytostructural dynamics of spreading and translocating cells. J. Cell Biol. 85, 127-136

Stoolman, L. M. (1989). Adhesion molecules controlling lymphocyte migration. Cell 56,907.

Taylor, R. B., Duffus, W. P. H., Raff, M. C. and de Petris, S. (1971). Redistribution and pinocytosis of lymphocyte surface immunoglobulin molecules induced by anti-immunoglobulin antibody. Nature, new Blol. 233, 225-229.

TrowbridGe, I. S., Legley, J., Schult'e, R., Hyman, R. and Trotthe, J
1982). Biochemical characterization and cellular distribution of a polymorphic, murine cell-surface glycoprotein expressed on lymphord tissues. Immunogenetzcs 15, 299-312.

Turner, C. E. AND Shotron, D. M. (1989). Effects of capping on the non-ionic detergent solubility of rat thymocyte glycoproteins Eur. Cell Biol 50,324-332.

Varlliev, J M., Gelfand, I. M., Domnina, L V., Dorfman, N. A. and Pletyushrina, O. Y. (1976) Active cell edge and movements of concanavalin A receptors of the surface of.epithelial and fibroblastic cells. Proc. natn. Acad. Sci. U.S.A. 73, 4085-4089.

WANG, Y,-L. (1985). Exchange of actin subunits at the leading edge of living fibroblasts. possible role of treadmilling. J Cell Biol. 101, $597-602$

WebB, W. W. AND Gross, D. (1986). Patterns of individual molecular motions deduced from fluorescent image analysis: In Applucations of Fluorescence in the Biomedical Scrences (ed. Taylor, D. L. et al.), pp. 405-422. Alan R Liss, NY

WODA, B A. AND MCFADDEN, M. L. (1983). Ligand-induced association of rat lymphocyte membrane proteins with the detergent-insoluble lymphocyte cytoskeletal matrix. J. Immun. 131, 1917-1919.

Wolffe, E. J., Gaube, W. C., Peifrey, C. M., Holland, S. M., Steingerg, A. D. AND Augurt, J. T. (1990). The cDNA sequence of mouse Pgp-1 and homology to human CD44 cell surface antigen and proteoglycan core/link proteins. J. biol. Chem. 265, 341-347.

(Received 6 September 1990 - Accepted 31 October 1990) 
